\title{
Assessment of Static Mechanical Properties of Additively Manufactured Polylactic Acid (PLA) on Entry-Level FDM 3D Printer
}

\author{
CONSTANTIN-ROMICA STOICA*, RALUCA MAIER, ANCA ISTRATE, \\ ANDREI-CRISTIAN MANDOC
}

Romanian Research and Development Institute for Gas Turbine COMOTI, 220D Iuliu Maniu Av., 061126, Bucharest, Romania

\begin{abstract}
The paper presents the study on the static mechanical properties of PLA (Polylactic Acid) produced with entry-level additive technologies using three printing directions. During the experimental work were tested a total of 15 "dog bone" ASTM D638-14 standard specimens made from additively manufactured polymer (PLA) through FDM (Fused Deposition Modelling) technique, where the material and rectilinear pattern infill geometry and infill percentage of $100 \%$ were constant and the printing orientation was varied. Usually technical data sheets that are delivered by filament materials producers include the most satisfactory data which are valid for only one specific printing direction. The printing direction is deliberately selected, in such way that the best material characteristics are achieved. In addition to this matter, as the additive manufacturing market grew significantly in the past couple of years, the filament production market showed a consequential growth. The aftermath of this expansion had a direct impact towards the quality and costs of the filaments used for $3 D$ printing, in order to satisfy both the low-end and high-end users. Therefore, in this frame, the present research provides entry-level additively manufactured PLA performances showing significant changes depending on the different printing directions and determine the build orientation influence on the mechanical properties, in the aim of providing aid for both mechanical designer and product manufacturer at the stage of the printed product mechanical properties.
\end{abstract}

Keywords: additive manufacturing, tensile testing, build orientation, PLA, FDM

\section{Introduction}

Additive manufacturing emerging technologies present a continuous growing and represents high interest for engineering due to undeniable major advantages related to increased automation level, low material usage and design freedom. The increased use of 3D printing as a learning tool and to generate functional end-use parts has brought out the need for a better understanding of the mechanical behavior of 3D printed parts and the development of analytical tools and design guidelines for engineers [1]. Currently used techniques include fused deposition modelling (FDM), one of the most used 3D printing techniques of thermoplastic polymers. This process is based on the deposition of semi-molten filaments to create an object as a composition of several overlapping layers; of which each layer is composed of filaments laid out according to a predetermined raster configuration. A significant advantage of FDM technology is the potential to create complex and specific shapes [2]. PLA (Polylactic Acid) is considered to be the most broadly researched and exploitted biodegradable aliphatic polyester, having prooved its potential in replacing several conventional petrochemical-based polymers for industrial applications, as well as showing leading characteristics as biomaterial in numerous applications in medicine $[3,4]$. In spite of being broadly researched, the data available is wildly scattered due to notable discrepancies between low-end and high-end available 3D printers and consequently, printing materials.

\footnotetext{
*email: romica.stoica@comoti.ro
} 
In an analysis of the physical and mechanical properties of PLA for a widerange of applications [5,6], it was discovered that data for young modulus varies between 0.35 and $3.5 \mathrm{GPa}$ which validates according to the range found in several studies that research the behaviour of PLA samples for tensile tests.

These studies differentiated themselves through different providers of the filament and 3D printers, although the same technology (FDM) was used with similar printing parameters $[2,7]$.

\section{Materials and methods}

\subsection{PLA filament sample preparation and tensile test experimental mechanical characterisation}

The material used for samples was PLA from Plusivo in black color, filament with $1.75 \mathrm{~mm}$ in diameter. The extrusion nozzle was $0.4 \mathrm{~mm}$ in diameter, the layer thickness was $0.2 \mathrm{~mm}$ for all layers. Specimen were printed at raster orientation angle of $45^{\circ}$ for the specimens in horizontal direction and $0^{\circ}$ for those fabricated in longitudinal and transversal direction. The building direction is shown in Figure 1.

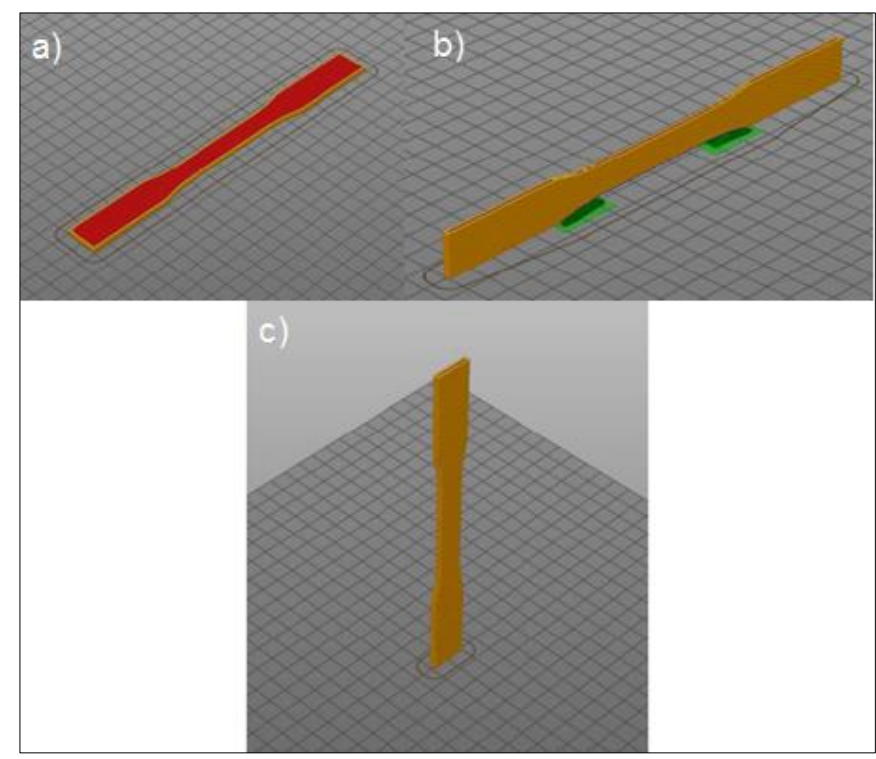

Figure 1. Build orientation of the samples a) horizontal

b) longitudinal and c) transversal

The tensile tests were performed according to ASTM D638-14 [8] with the samples printed in three directions. The printing directions are shown in Figure 1 and the sample dimensions are shown in Figure 2. The tensile testing machine was an Instron 8872 with a load cell of $25 \mathrm{kN}$. The testing speed was set to $5 \mathrm{~mm} / \mathrm{min}$, an Instron 2620-601 extensometer of $50 \mathrm{~mm}$ gauge length was used to determine the tensile strain. The specimens have an overall length bigger than the minimum specified $(200 \mathrm{~mm}$ instead of $165 \mathrm{~mm}$ ) in [8] because the clamping zone was enlarged to avoid failure in the clamping region.

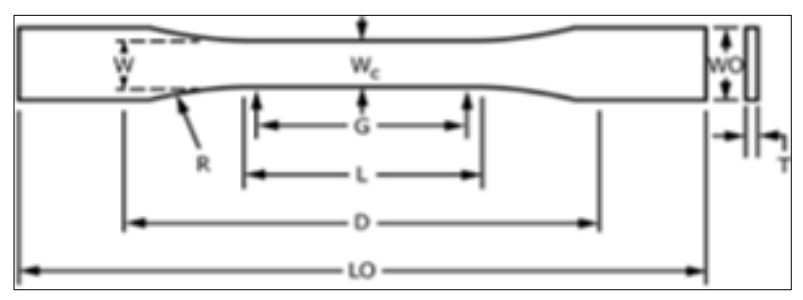

Figure 2. Sample geometry according to ASTM D638- 14 [8] 
Table 1. Tensile samples dimensions

\begin{tabular}{|c|c|c|}
\hline Dimensions according to ASTM D638-14 & $\begin{array}{c}\text { Value } \\
(\mathrm{mm})\end{array}$ & Tolerance (mm) \\
\hline W- Width of narrow section & 13 & \pm 0.5 \\
\hline L- Length of narrow section & 57 & +6.4 \\
\hline WO- Width overall & 19 & no maximum \\
\hline LO- Length overall & 165 & \pm 0.25 \\
\hline G- Gage length & 50 & \pm 5 \\
\hline D- Distance between grips & 115 & \pm 1 \\
\hline R- Radius of fillet & 76 & \\
\hline
\end{tabular}

The probes were manufactured on a Geetech A30 FDM machine using the PrusaSlicer2.0 preprocessing program with Expert Mode settings to control printing parameters. The samples were printed from the same filament spool with print jobs of 5 samples at the same time to avoid water absorption of the samples by reducing the total fabrication time.

Table 2. Main fabrication parameters

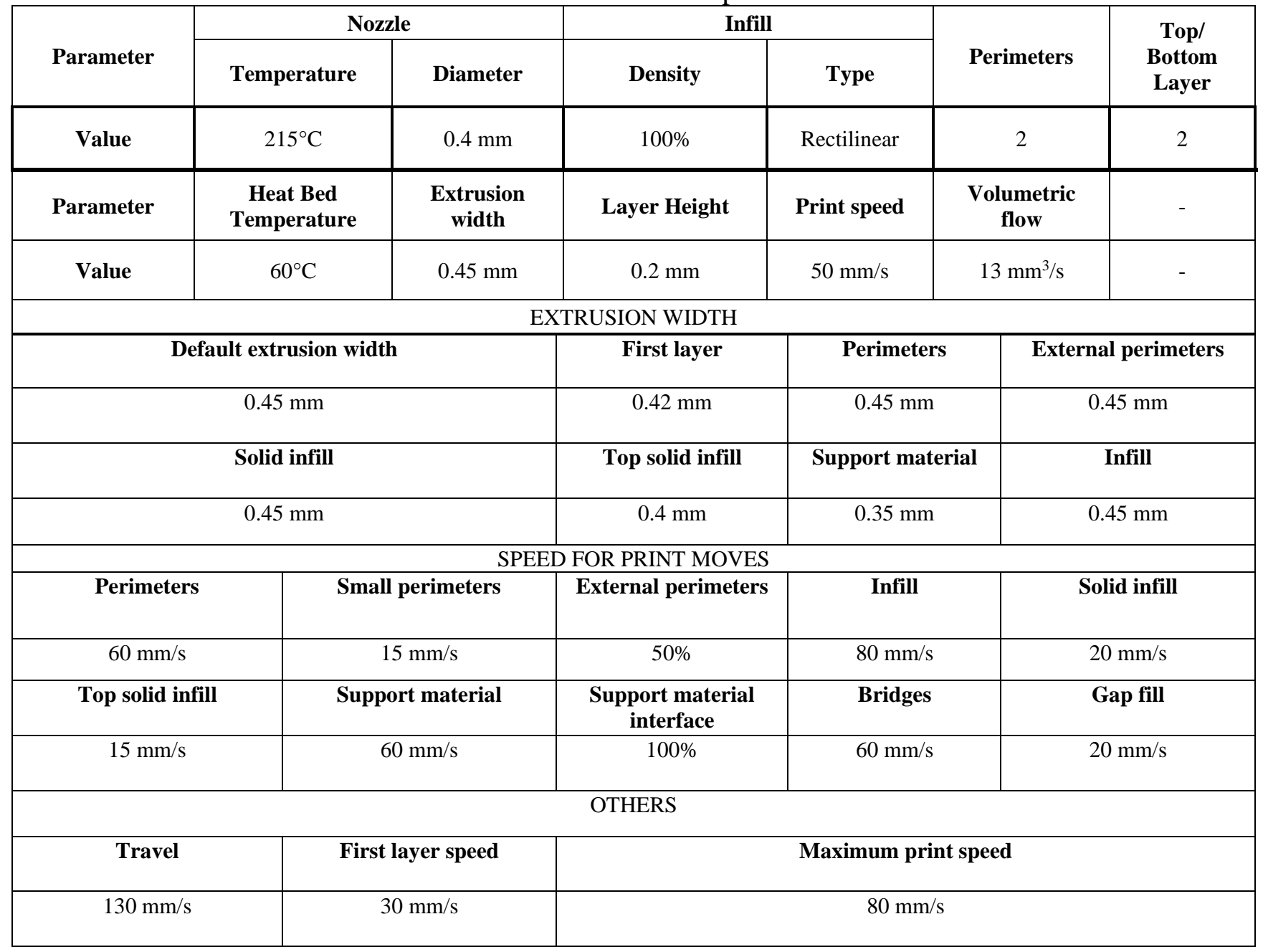

The samples were measured to evaluate the tolerances of the manufactured parts and all samples were in the limits prescribed by the tensile testing standard. Due to technical errors that occurred during 
the static test campaign, it was concluded that the data obtained for some samples did not meet the requirements to be validated, therefore a minimum of 3 samples were tested according to the standard [8].

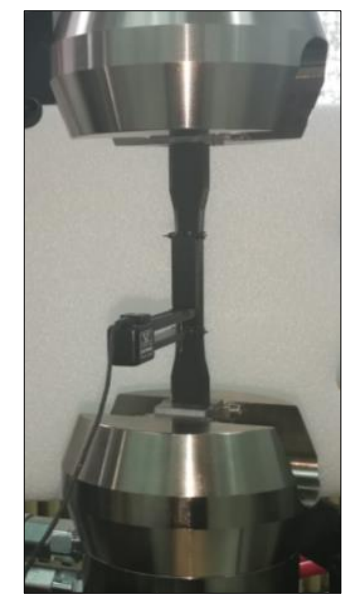

Figure 3. Sample mounted on the Instron 8872 (load cell of $25 \mathrm{kN}$ ) hydraulic testing machine with the strain gauge mounted before testing

\section{Results and discussions}

The stress-strain curves of all tested specimens are plotted in Figure 4, Figure 8, Figure 10, the straight lines indicate the Young modulus at a $0.2 \%$ offset. Both yield and ultimate tensile strength are represented by black dots. In Figure 4 we notice that for the longitudinal printing direction an ultimate tensile strength of approximately $35 \mathrm{MPa}$ is obtained, a value that exceeds the properties of an aluminium alloy series 1000, but lower than an alloy of series 2000 which has a yield strength to have a minimum value of $168 \mathrm{MPa}$. If we compare the specific stresses (MPa/kg), polylactic acid is only twice as weak as a 2000 series aluminium alloy, which is an aviation alloy used in shell panels and other parts with a structural role on an aircraft. The elongation values are within the values for fragile materials, the material has almost no plastic deformations, the maximum value of elongation at complete failure is $3 \%$ and an average value for elongation at break, for this direction, is around 2.5\% except for the sample L5 which shows a smaller elongation at break. From all the tests performed in all printing directions, the longitudinal direction showed the highest strength values, which are found in Table 3.

It is observed that the modulus of elasticity has values that depend on the printing direction, and as expected the highest value of the modulus of elasticity $E$ is on the longitudinal direction where most of the fibres are oriented in the direction of the tensile load. how are they oriented for the horizontally built specimens?

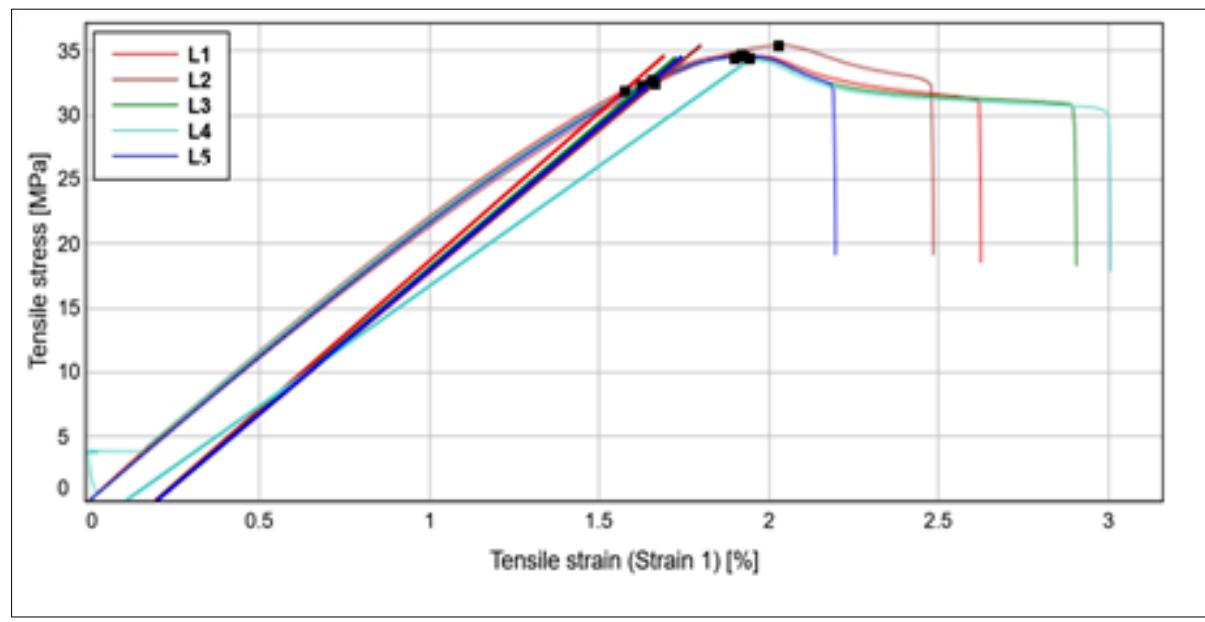

Figure 4. Stress-strain curves of the longitudinal built samples 


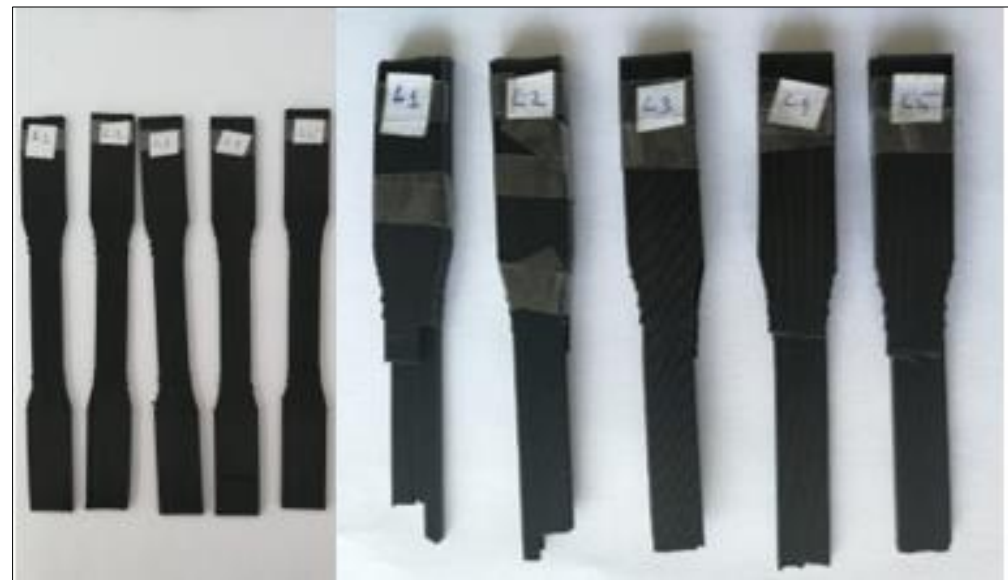

Figure 5. Longitudinal samples before and after testing

The breaking direction for this orientation is predominant following the direction perpendicular to the load as can be seen in Figure 5. For L1 and L2 specimens the fracture plane is not cutting thought a certain orientation from the applied load, this may be caused by imperfections in the area like pores and inclusions from the printing process. The breaking occurs in the lower side of the narrow section because of the stress concentration in the radius due to the printing direction poor resolution of producing the part, this can be seen in Figure 5 in the fact that the radius is not smooth, aspect reported also in [7].

Table 3. Mechanical properties for the longitudinal (L) specimens

\begin{tabular}{|c|c|c|c|}
\hline \multirow{2}{*}{ Sample } & \multicolumn{3}{|c|}{ Parameter } \\
\cline { 2 - 4 } & $E[\mathrm{MPa}]$ & $R_{\mathrm{m}}[\mathrm{MPa}]$ & $\begin{array}{c}\text { Yield strength } \\
(0.2 \%)[\mathrm{MPa}]\end{array}$ \\
\hline L1 & 2313.00 & 34.68 & 31.93 \\
\hline L2 & 2212.65 & 35.47 & 32.48 \\
\hline L3 & 2257.72 & 34.53 & 32.30 \\
\hline L4 & 1870.20 & 34.45 & 34.36 \\
\hline L5 & 2238.40 & 34.61 & 32.67 \\
\hline
\end{tabular}

The horizontal $(\mathrm{H})$ direction printed samples exhibit higher mechanical strength compared to transversal and lower than longitudinal ones, the difference between the longitudinal and horizontal direction being due to the orientation of the fibres which are no longer as numerous in the direction of the load. The filling of the specimens being done in the directions $+/-45^{\circ}$ as in Figure 6 . The breaking of the specimens is done predominantly in the direction of $45^{\circ}$ represented in Figure 7.

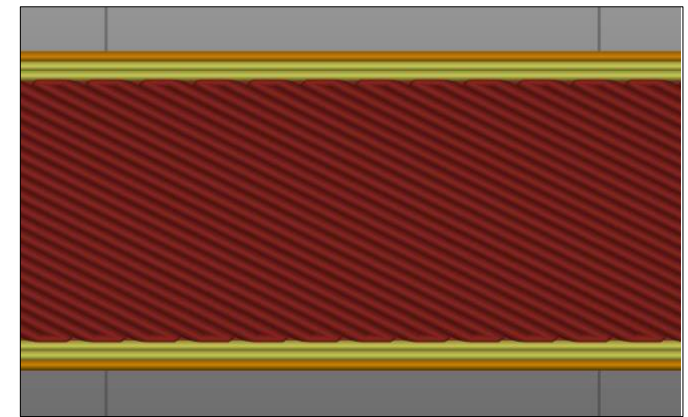

Figure 6. Detail with the rasper angle of the horizontal $(\mathrm{H})$ specimens 


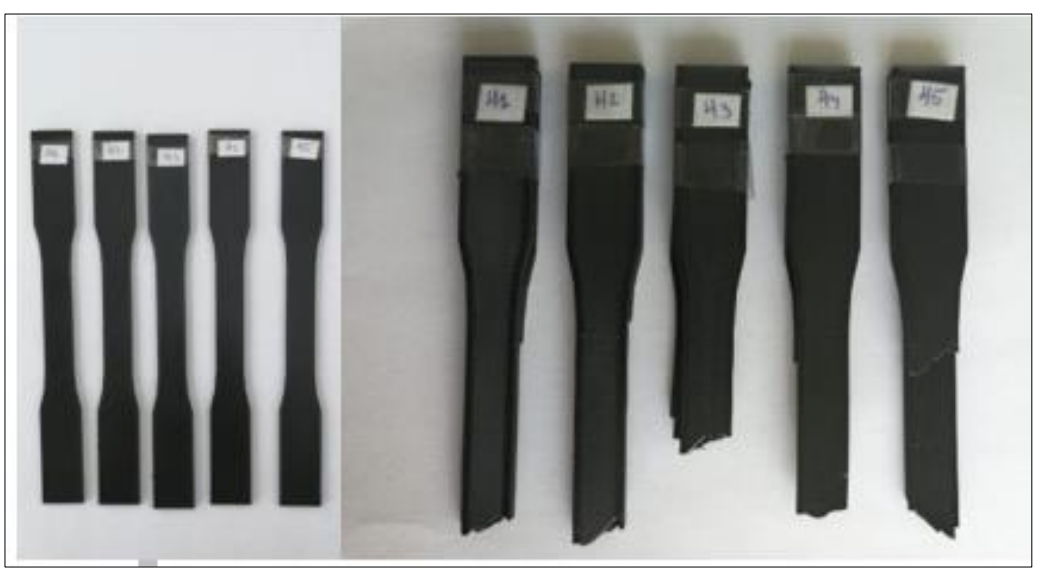

Figure 7. Horizontal $(\mathrm{H})$ specimens fracture direction why do you use this abbreviation if it is not used in text! specimens

Figure 8 shows the stress-strain curves for the specimens printed in the horizontal direction $(\mathrm{H})$, it can be observed that the material has a fragile character of rupture because the elongation at break does not exceed $2 \%$, while the ultimate tensile strength varying between 18 and $21 \mathrm{MPa}$. The fracture plane angle is heavily influenced by the orientation of the fibres, the yield being made in planes parallel to the orientation of the fibres. The contribution of the three exterior perimeters that are along the load direction can create optimistic values of the maximum stress, thus the values presented are slightly higher than those with no perimeters. In [9] the authors also investigated the influence of shell thickness (encompassing the perimeter in the horizontal plane and the solid layers forming the bottom and the top) and found that the shell thickness (i.e., the number of layers) had a large influence on the tensile strength of the samples.

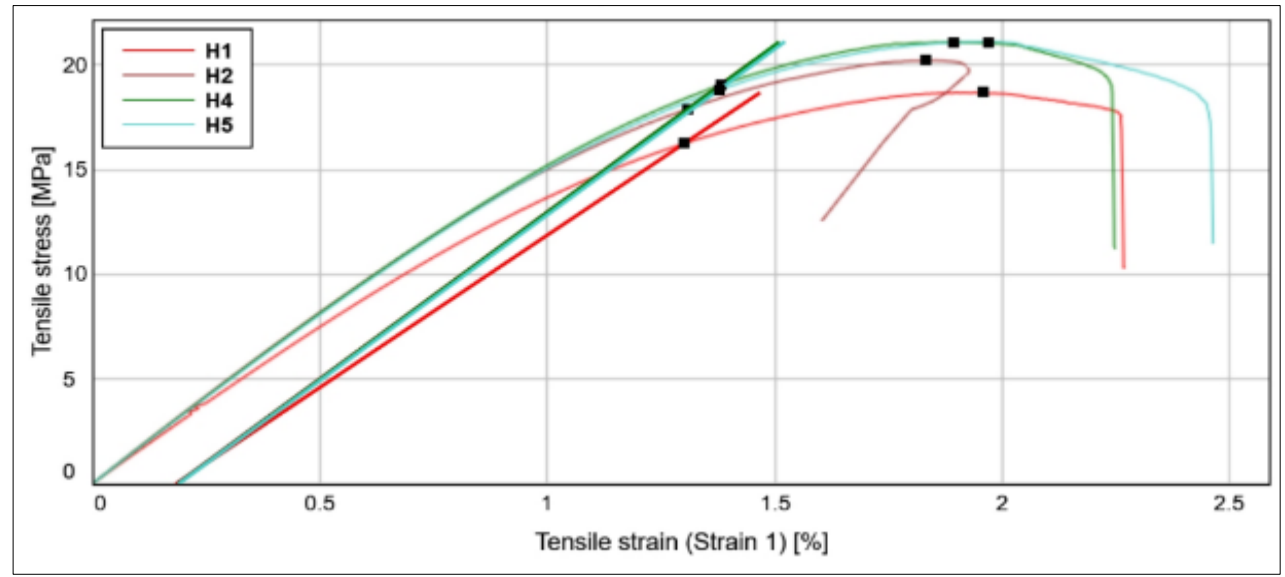

Figure 8. Stress-strain curves for the horizontal built samples

Table 4. Mechanical properties for the horizontal $(\mathrm{H})$ specimens

\begin{tabular}{|c|c|c|c|}
\hline \multirow{2}{*}{ Sample } & \multicolumn{3}{|c|}{ Parameter } \\
\cline { 2 - 4 } & $E[\mathrm{MPa}]$ & $R_{\mathrm{m}}[\mathrm{MPa}]$ & Yield strength $(0.2 \%)[\mathrm{MPa}]$ \\
\hline H1 & 1454.32 & 18.68 & 16.23 \\
\hline H4 & 1596.64 & 21.10 & 19.03 \\
\hline H5 & 1583.90 & 21.11 & 18.81 \\
\hline
\end{tabular}

Stress-strain curves for transversal built samples tested in static regime are reported in Figure 10. Mechanical properties for the transversal $(\mathrm{T})$ specimens given in Table 5 clearly show a significant lower mechanical strength compared to horizontal and longitudinal direction printed samples, due to the lack of fibres orientated in the loading direction. Likewise, the adhesion between the layers is lower for 
transversal boundaries since slight pressure is applied from the nozzle to the melted filament while the filament is being extruded.

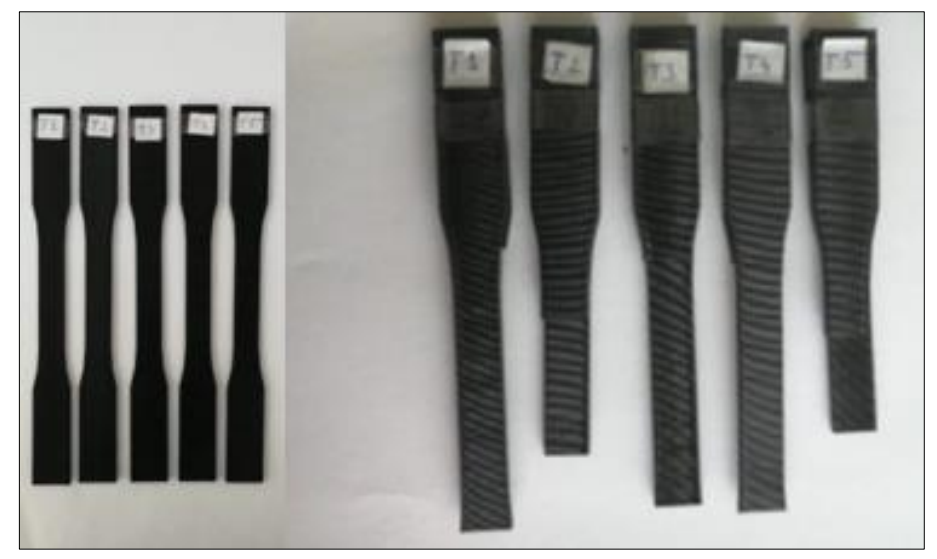

Figure 9. Transversal (T) samples before and after testing

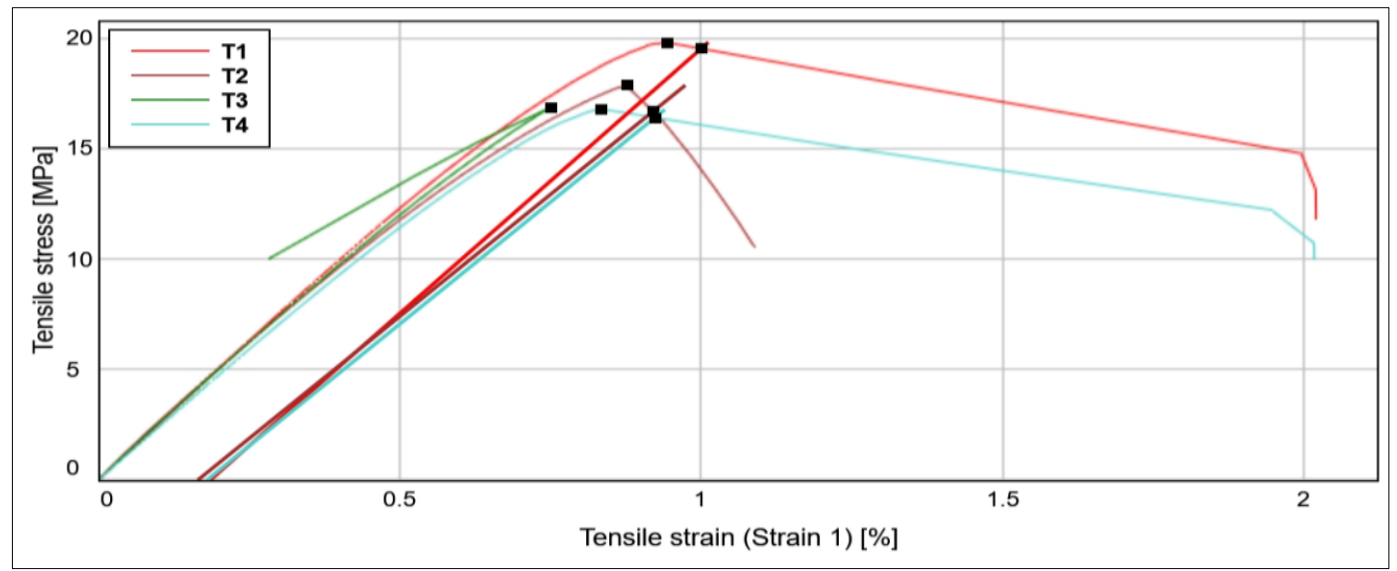

Figure 10. Stress-strain curves for transversal built samples

Table 5. Mechanical properties for the transversal $(\mathrm{T})$ specimens

\begin{tabular}{|c|c|c|c|}
\hline \multirow{2}{*}{ Sample } & \multicolumn{3}{|c|}{ Parameter } \\
\cline { 2 - 4 } & $E[\mathrm{MPa}]$ & $R_{\mathrm{m}}[\mathrm{MPa}]$ & Yield strength $(0.2 \%)[\mathrm{MPa}]$ \\
\hline $\mathrm{T} 1$ & 2398.23 & 19.81 & 19.52 \\
\hline $\mathrm{T} 2$ & 2210.12 & 17.86 & 16.66 \\
\hline $\mathrm{T} 3$ & 2308.83 & 16.87 & - \\
\hline T4 & 2208.56 & 16.77 & 16.40 \\
\hline
\end{tabular}

The research study performed on PLA mechanical properties in static regime using steady tensile rate mechanical tests showed that the polymeric material obtained by additive production is an orthotropic, brittle material with relatively good mechanical strength [2]. Table 6 below display the average mechanical properties of PLA and Table 7 a comparison with data from the literature regarding the influence of perimeter usage [5].

The average mechanical properties of PLA are listed in Table 6 for comparison with the values obtained from the experimental campaign. If we compare the values obtained from Table 7 which represent average values obtained from the literature we can conclude that an ultimate tensile strength was obtained with low to medium value depending on the printing direction. The elongation at break is towards the lower limit of the values obtained in several works $[1,2,5]$. 
Table 6. PLA properties variations [5]

\begin{tabular}{|c|c|c|}
\hline Property & Units & Variation interval \\
\hline Density $(\rho)$ & $\mathrm{g} / \mathrm{cm}^{3}$ & $1.21-1.25$ \\
\hline UTS $(\sigma)$ & $\mathrm{MPa}$ & $21-60$ \\
\hline Young Modulus $(\mathrm{E})$ & $\mathrm{GPa}$ & $0.35-3.5$ \\
\hline Strain at break $(\varepsilon)$ & $\%$ & $2.5-6$ \\
\hline Temperature of glass transition $\left(T_{\mathrm{g}}\right)$ & ${ }^{\circ} \mathrm{C}$ & $45-60$ \\
\hline Melting Point $\left(T_{\mathrm{p}}\right)$ & ${ }^{\circ} \mathrm{C}$ & $150-162$ \\
\hline
\end{tabular}

Table 7. Comparison with data from the literature

\begin{tabular}{|c|c|c|c|c|c|c|}
\hline \multirow[b]{2}{*}{ Paper } & \multirow[b]{2}{*}{$\begin{array}{l}\text { Number of } \\
\text { perimeters }\end{array}$} & \multicolumn{4}{|c|}{ Experimental values } & \multirow[b]{2}{*}{ Observation } \\
\hline & & $\begin{array}{c}\text { UTS } \\
{[\mathrm{MPa}]}\end{array}$ & $\begin{array}{l}\text { Young } \\
\text { modulus } \\
{[\mathrm{MPa}]}\end{array}$ & $\begin{array}{l}\text { Raster angle } \\
\text { orientation }\left[{ }^{\circ}\right]\end{array}$ & $\begin{array}{c}\text { Build } \\
\text { orientation }\end{array}$ & \\
\hline \multirow{3}{*}{$\begin{array}{l}\text { Current } \\
\text { study }\end{array}$} & 0 & 35 & 2200 & 0 & Longitudinal & \multirow{3}{*}{ Rounded average values } \\
\hline & 2 & 34 & 2200 & 45 & Horizontal & \\
\hline & 0 & 18 & 2300 & 90 & Transversal & \\
\hline [1] & 3 & 46.18 & 973 & 0 & Horizontal & Estimated from Figure 17 \\
\hline \multirow{2}{*}[2]{} & 2 & 43 & 2600 & $0 / 90$ & Horizontal & \multirow{2}{*}{ Extracted from Figure 6} \\
\hline & 2 & 45 & 2800 & 45 & Horizontal & \\
\hline \multirow{3}{*}{ [10] } & 1 & 45 & N/A & 0 & Horizontal & \multirow{3}{*}{ Extracted from Figure $7 \mathrm{c}$ ) } \\
\hline & 1 & 35 & N/A & 45 & Horizontal & \\
\hline & 1 & 25 & N/A & 90 & Horizontal & \\
\hline
\end{tabular}

The modulus of elasticity obtained for the tested specimens is positioned at the level of medium to high values compared to other studies. The differences between the values obtained in this work and other works can be given by: printing temperature, printing speed, filament manufacturer's recipe and quality but it is not limited to these aspects. More specifically, the study of Grasso et. al. [2], where an infill density of $40 \%$ and a number of two perimeters were used, and Rajpurohit et. al. [10], where an infill density of $100 \%$ and only one perimeter were used, present high discrepancies in terms of results collected irefor samples with $45^{\circ}$ raster orientation. But, when compared to this study, the UTS reported in [10] is $34 \mathrm{MPa}$, similar to $35 \mathrm{MPa}$ which was reported in this work. Both studies, [2, 10], utilized more accurate and high-end user 3D printers, but none of them specifiy the filament manufacturer. After consulting their work, it was clear that although, the infill percentage has usually an influence, it was not the case in this paper. Neither was the number of perimeters. The only data that is different in all three of the studies, is the material, which can differ in several aspects like quality of manufacturing and storage (humidity, dust, receipt, etc.) given the fact that most of the parameters are similar in all three studies. According to the data collected in the research paper [10], it can be concluded that it is preffered to have a smaller layer height with a larger raster extrusion width, one of the reasons being that the layers have a larger bonding area. This backs up the data obtained in the present study, given the fact that the minimum raster extrusion width was selected $(450 \mu \mathrm{m})$. The fracture plane orientation is perpendicular to the fibre orientation for the longitudinal direction and parallel for the probes fabricated in horizontal and transversal direction. In other studies, the raster orientation was varied [1, 2, 10]. For some directions the values are slightly higher than those presented for the transversal direction but the samples were printed flat on the heat bed with perimeters around the specimen, thus some layers were oriented parallel to the load yielding optimistic results in terms of Young modulus and strength, because of the contribution of the perimeters layer that are oriented in the load direction. Thus, for parts built on the $\mathrm{Z}$ direction (transversal) with $100 \%$ infill are not influenced by the raster angle orientation, instead the layer adhesion is more important for this fabrication direction. For the horizontal samples, two perimeters were added due to technological reasons. 


\section{Conclusions}

The present research study performed on PLA mechanical properties in static regime using steady tensile rate mechanical tests showed that the polymeric material obtained by additive production is an orthotropic, brittle material with relatively good mechanical strength. Upon entry-level or qualified additive technologies used, as well as a wide range of printing parameters (e.g. material purity, filament thickness, infill geometry pattern and ratio, printing orientation, etc) mechanical performances exhibits significant changes. In this frame, the present research work provides entry-level additively manufactured PLA standard test specimens performances upon printing orientation whereas material and rectilinear pattern infill geometry, infill percentage of $100 \%$ were constant. Results showed significant changes in mechanical properties depending on different printing directions, all data being in the low middle range of PLA properties variations [5]. The elongation at break is towards the lower limit of the values obtained in several works $[1,2,5]$ whereas the modulus of elasticity obtained for the tested specimens is positioned at the level of medium to high values compared to other studies. The fracture plane orientation is perpendicular to the fibre orientation for the longitudinal direction and parallel for the probes fabricated in horizontal and transversal direction, as expected. The present research results, obtained on entry-level additive technology manufactured PLA, were reported in the aim of providing aid for both mechanical designer and product manufacturer with respect to mechanical performances of printed products, in the frame of printing parameters variation. In conclusion, polylactic acid can be used even for light mechanically stressed parts, and for non-structural parts it can be a competitive replacement of metal especially for complex geometry parts, but a more thorough investigation is required to determine the material properties on all axis and while several technological parameters have a strong influence on the behaviour of the final product, thus a rigorous control of fabrication is required.

\section{References}

1. D. FARBMAN, C. MCCOY, "Materials Testing of 3D Printed ABS and PLA Samples to Guide Mechanical Design," in Proceedings of the ASME International Manufacturing Science and Engineering Conference MSEC, Blacksburg, Virginia, USA, 2016

2. M. GRASSO, L. AZZOUZ, P. RUIZ-HINCAPIE, M. ZARRELLI, G. REN, "Effect of temperature on the mechanical properties of 3D-printed PLA tensile specimens," Rapid Prototyping Journal, vol. 24, no. 8, pp. 1337-1346, 2018.

3. A. JARDINI, R. M. FILHO, M. SAVIOLI LOPES, "Poly (Lactic Acid) Production for Tissue Engineering Applications," Procedia Engineering, vol. 42, pp. 1402- 1413, 2012

4. R. DRUMRIGHT, P. GRUBER, D. HENTON, "Polylactic Acid Technology," Advanced Materials, vol. 12, pp. 1841- 1846, 2000.

5. S. FARAH, "Physical and mechanical properties of PLA, and their functions in widespread applications - A comprehensive review," Advanced Drug Delivery Reviews, pp. 367-392, 2016.

6. K. VAN DE VELDE, P. KIEKENS, "Biopolymers: overview of several properties and consequences on their applications," Polymer Testing, vol. 21, no. 4, pp. 433-442, 2002

7. T. LETCHER, W. MEGAN, "Material Property Testing of 3D-Printed Specimen in PLA on an EntryLevel 3D Printer," in Proceedings of the ASME 2014 International Mechanical Engineering Congress and Exposition. Volume 2A: Advanced Manufacturing, Montreal, 2014

8.*** ASTM D638-14, "Standard Test Method for Tensile Properties of Plastics," American Society for Testing and Materials, 2014

9. T. PEPELNJAK, A. KARIMI, A. MACEK, N. MOLE, "Altering the Elastic Properties of 3D Printed Poly-Lactic Acid (PLA) Parts by Compressive Cyclic Loading," Materials, vol. 13, p. 4456, 2020

10. S. R. RAJPUROHIT, H. K. DAVE, "Effect of process parameters on tensile strength of FDM printed PLA part," Rapid Prototyping Journal, vol. 24, no. 8, pp. 1317-1324, 2018.

Manuscript received: 22.02 .2021 